odor which could be reliably used as the discriminative stimulus by these animals, the solvent was eliminated and only the food-odor stimulus was evaluated.

The intensity of the food-odor stimulus was increased by increasing the pellet reward from four to six to determine if more stable performance might be obtained from the fourth animal. This increase resulted in significant discrimination by all animals. Also, the six-pellet reward resulted in a clearer differentiation between performances, with and without the food odor cues, than did the four-pellet reward. As can be seen from Fig. 1B, a dramatic shift in performance occurs when the odor stimuli are controlled by baiting both cups but only pushing in the appropriate one after the animal has made the correct choice (pellets absent) as compared with blocks of trials where only the correct cup was baited and inserted (odor stimulus present). This effect was easily replicated with the additional finding that the rat with the poor performance on early trials was now able to consistently perform at a significant Ievel. Finally, when the reward size was reduced by one pellet for each succeeding 12-trial block (Fig. 1C), three animals continued their good performances with only a single-pellet reward. A fourth animal was unable to discriminate with fewer than four pellets.

This study demonstrates, contrary to most earlier findings, that rats can learn to make the correct discrimination in a $\mathrm{T}$-maze to odor cues. Once the animals learn this discrimination it easily generalizes to other olfactory stimuli. Further, it is apparent from this study that the rat is capable of performing a discrimination task successfully with the odor from only a very small quantity ( $45 \mathrm{mg}$ ) of dried food contained in a single Noyes pellet.

Studies concerned with the manipulation of the amount of reward should be seriously concerned with the control of odor stimuli. In many of these experiments, manipulations in reward magnitude result in concomitant manipulations in the intensity of food odor which may then serve as a discriminative stimulus. The fact that rats can perform at a significant level with only the odor of one dry $45-\mathrm{mg}$ food pellet diluted with a large volume of background air serving as a discriminative stimulus clearly shows that odor controls are necessary. With this degree of sensitivity it is not enough to assume that the presence of extraneous food particles and the lack of an established odor gradient are sufficient to minimize the effects of odor stimuli serving as discriminative stimuli.

\section{REFERENCES}

HONZIK, C. H. The sensory basis of maze learning in rats. Comparative Psychology Monograph,
$1936,13,1$

LIGGETT, J. R. An experimental study of the olfactory sensitivity of the white rat, Genetic Psychology Monograph, 1928, 3, 1-64.

LONG, C. J., \& TAPP, J. R. An apparatus for the assessment of the reinforcing properties of odors in small animals. Journal of the Experimental Analysis of Behavior, 1968, 11, 49-51.

LUDVIGSON, H. W., \& SYTSMA, D. The sweet smell of success: Apparent double alternation in the rat. Psychonomic Science, 1967, 9,
283-284.

MILLER, S. D., \& ERICKSON, R. P. The odor of taste solutions. Physiological Behavior, 1966, 1, 145-146.

VINCENT, S. B. The white rat and the maze problem: II. The introduction of an olfactory control. Joumal of Animal Behaviour, 1915, 5, $140-157$.

WATSON, J. B. Kinaesthetic and organic sensations: Their role in the reactions of the white rat. Psychological Review Monograph, $1907,8,1-100$.

\title{
Social dominance in gerbils and hamsters
}

ROBERT BOICE, University of Missouri, Columbia, Mo. 65201, DANIEL HUGHES, and CHRISTINE J. COBB, Ohio University, Athens, Ohio 45701

Dominance orders in four groups of gerbils (Meriones unguiculates) and four groups of hamsters (Mesocricetus auratus) were measured in two conventional tests. Both species evidenced reliable rank orders in a majority of group-test combinations. Water competition rankings were associated with obvious aggression and were positively correlated with body weight. In the gerbils, tunnel competition rankings were not accompanied by overt aggression and were negatively correlated with body weight and uncorrelated with water dominance orders. In the hamsters, tunnel dominance orders were mediated by overt aggression and were positively correlated with both body weight and water dominance orders.

Social dominance is typically defined in terms of stable orders of precedence within animal groups (e.g., Allee, 1951). Because precedence orders are reflected in diverse behaviors such as food-getting and copulation, different tests of dominance may not be measures of the same general trait. For example, Lindzey et al (1966) found a negative correlation between tunnel dominance and food competition in Mus. Similarly, one species may show reliable hierarchies in a measure which does not produce stable rankings in another species (Candland \& Bloomquist, 1965). The present study compared dominance in possession of a water spout and in removing a competitor from a tunnel in two genera of the family Cricetidae. One rodent, the gerbil, is unusually nonaggressive in comparison to the second, the hamster.

The selection of dominance tests was influenced by specific characteristics of the rodents. Spontaneous aggression (Baenninger, 1966) was excluded because gerbil groups established for more than 3 days showed overt aggression only when thirsty or hungry and in pursuit of water or food. Water competition was substituted for the more conventional test of food competition (Becker \& Flaherty, 1968) since hamsters are less likely to persist in competition to fill their food pouches than to drink water. Gerbils do consume drinking water with apparent avidity (Boice \& Arledge, 1968).

\section{SUBJECTS}

Sixteen male gerbils /Meriones unguiculates) were obtained from Tumblebrook Farms and 16 male golden hamsters (Mesocricetus auratus) were obtained from E. G. Steinhilber \& Co. All were maintained in plastic cages $(33 \times 45 \times 30 \mathrm{~cm})$ with wire tops and substrates consisting of two parts dried wood chips and one dried clay. Water was presented, when appropriate, from inverted cylinders with metal drinking spouts. Maintenance food was Wayne Breeder Blox.

Original assignment to intraspecific experimental groups of four was made on the basis of age. Groups 1, 2, and 3 for each species consisted of males of $71,98,126$, and 200 days of age at the start of testing. Group 4 consisted of four immature males of 36 days of age. The occurrence of respiratory ailments in the youngest hamsters following shipment arrival forced the substitution of a heterogeneous age group for the young-hamster group.

Testing began with 7 days of tunnel pretraining in which the gerbils learned to run from one endbox of a tunnel ( $45 \times 3-6 \mathrm{~cm}$, according to girth of the $\mathrm{Ss}$ ) to the opposite end to obtain 5-min access to unshelled sunflower seeds. Two such pretraining trials were run daily with goal sites and start sites switched randomly between ends. Water but not food was available in home cages on days of tunnel experimentation. 
Table 1

Stability of Dominance Orders

\begin{tabular}{|c|c|c|c|c|}
\hline \multirow[b]{2}{*}{ Group } & \multicolumn{2}{|c|}{ Gerbils } & \multicolumn{2}{|c|}{ Hamsters } \\
\hline & $\begin{array}{l}\text { Tunnel } \\
\mathbf{W}-\mathbf{r}_{\text {Sav }}\end{array}$ & $\begin{array}{c}\text { Water } \\
\text { W-ISav }\end{array}$ & $\begin{array}{l}\text { Tunnel } \\
\mathbf{W}-{ }^{-} \text {Sav }\end{array}$ & $\begin{array}{c}\text { Water } \\
\text { W- r Sav }\end{array}$ \\
\hline $\begin{array}{l}1 \\
2 \\
3 \\
4 \\
\end{array}$ & $\begin{array}{l}0.72-0.70^{* *} \\
0.38-0.34^{* *} \\
0.60-0.57^{* *} \\
0.16-0.10\end{array}$ & $\begin{array}{l}0.70-0.68^{* *} \\
0.81-0.80^{* *} \\
0.21=0.15^{*} \\
0.45-0.41^{* *}\end{array}$ & $\begin{array}{l}0.62-0.59^{* *} \\
0.20-0.15 \\
0.77-0.75^{* *} \\
0.21-0.15\end{array}$ & $\begin{array}{l}0.14-0.08 \\
0.63-0.60^{* *} \\
0.46-0.46^{* *} \\
0.62-0.59^{* *}\end{array}$ \\
\hline
\end{tabular}

Table 2

Correlations Between Body Weight, Tunnel Dominance and Water Dominance

\begin{tabular}{|c|c|c|}
\hline \multirow[b]{2}{*}{$\begin{array}{l}\text { Body Weight } \\
\text { Water Competition }\end{array}$} & Water Dominance & Tunnel Dominance \\
\hline & $\mathrm{r}=+.42$ & $\begin{array}{l}r=-.32 \\
r=-.05\end{array}$ \\
\hline $\begin{array}{l}\text { Body Weight } \\
\text { Water Competition }\end{array}$ & $r=+.48$ & $\begin{array}{l}r=+.65 \\
r=+.49\end{array}$ \\
\hline
\end{tabular}

Fifteen test days followed in which tunnel dominance was assessed by pairing each member of a group with each other member of a group once a day, in random order. In each test trial the two combatants were placed at opposite ends of the tunnel; the rodent forcing the other out of the tunnel was considered dominant on that trial. These test trials did not include food reinforcement since placement of seeds in either endbox would have been complicated by the presence of animals who had not yet entered the tunnel or the joint presence of the winner and loser. In an attempt to prevent extinction of the tunnel-running response, all $S s$ received individual reinforced trials, as in pretraining, at the conclusion of paired test trials.

For the next 15 days, food was supplied freely in the home cage but water availability was limited to $15 \mathrm{~min}$ per day. Water dominance was judged daily according to the order of drinking and the outcomes of aggressive efforts to gain possession of the water spout. Body weights were obtained every other day throughout the experiment.

\section{RESULTS}

Table 1 shows the calculated stability produced by the two tests of dominance. The Kendall coefficient of concordance $(W)$ reflects the extent of agreement between daily rank orders. This coefficient is also expressed as an average rank intercorrelation ( $\mathrm{r}_{\mathrm{Sav}}$ ) to permit a test of the hypothesis of no difference in rankings over days (Siegel, 1956).

In the gerbil groups, only Group 4 with its immature members failed to show significantly stable rankings in the tunnel test. All gerbil groups showed statistically significant agreement between rankings over days in the water dominance test. Body weights in gerbils were positively related to water dominance but negatively related to tunnel dominance (Table 2). Accordingly, the two tests of dominance were essentially unrelated.

In the hamsters, rank orders were significantly stable in three of four groups in terms of water competition and in two of the four groups in terms of tunnel competition (Table 1). Both tests of dominance were positively related to body weights in the hamsters (Table 2). The relation between mean rankings of $\mathrm{Ss}$ in both tests was positive.

\section{DISCUSSION}

Assuming that intraspecies competition is an important dimension of species survival (Thiessen et al, 1968), the resolution of that competition into stable orders might be expected even in domesticated species. Both the gerbils and hamsters of the present study showed evidence in most groupings of stable dominance orders as defined by two conventional tests. A cross-species comparison of the performances in the two tests raises questions concerning the definition of dominance.

Dominance as defined in terms of the competitive drinking situation is clearly not related to dominance as defined in the tunnel situation for the gerbils. As noted by Lindzey et al (1968), the consummatory situation can provide more freedom of movement and more opportunity for aggression than does a tunnel situation. Higher ranked gerbils in the water test would frequently scratch at the faces or climb over the backs of opponents when gaining access to the water spout. Water test bouts were accompanied by heightened activity and squealing. In contrast, the gerbils in the tunnel situation showed no evidence of excitement or overt aggression. Some tunnel encounters involved head butting but most trials ended with the retreat of one gerbil after momentary contact. Gerbils which were primarily submissive in the water test were often rated dominant in the tunnel test. Gerbil A of Group 4, for instance, rarely showed aggressive behavior and would immediately give up the water tube if approached by an opponent. In the tunnel test, however, he obtained the highest rating of all 16 gerbils. He simply lay in the tunnel and passively resisted the pushing of his opponent. After his opponent retreated from the tunnel, he then completed his run through the tunnel. Aggressive behaviors were least apparent in the group of young gerbils. In initial trials of the water test, they often queued to the water tube and showed the pushing behaviors characteristic of older gerbils only in terminal trials.

Dominance as defined by both tests appears to be basically equivalent for the hamsters. The hamsters, in contrast to the gerbils, used aggressive behaviors such as biting and clawing in both the water and tunnel situations. Body weight (or age) was positively correlated with both measures.

Grant \& Chance (1958) note that dominance should be defined in terms of behaviors which precede and are basic to orders of precedence and not necessarily in terms of the precedence orders themselves. If a test does not involve behaviors basic to the resolution of social encounters (e.g., aggression), as may have been the case with the gerbils in the tunnel test, then perhaps that test should not be labeled a dominance measure for that species.

\section{REFERENCES}

ALLEE, W. C. Cooperation among animals. New York: Henry Schuman, 1951.

BAENNINGER, L.P. The reliability of dominance orders in rats. Animal Behaviour, 1966, 14, 367-371.

BECKER, G., \& FLAHERTY, T. B. Group size as a determinant of dominance hierarchy stability in the rat. Journal of Comparative \& Physiological Psychology, 1968, 66, 443-445.

BOICE, R., \& ARLEDGE, J. G. Water requirements of gerbils and kangaroo rats in the laboratory. Psychological Reports, 1968, 23, 1063-1069.

CANDLAND, D. K., \& BLOOMQUIST, D. W. Interspecies comparisons of the reliability of dominance orders. Journal of Comparative \& Physiological Psychology, 1965, 66, 443-445.

GRANT, E. C \& CHANCE, M. R. A. Rank order in caged rats. Animal Behaviour, 1958, 6, 92-111.

LINDZEY, G., MANOSEVITZ, M., \& WINSTON, $H$. Social dominance in the mouse. Psychonomic Science, 1966, 5, 451-452.

THEISSEN, D. D., FRIEND, H. C., \& LINDZEY, G. Androgen control of territorial marking in the Mongolian gerbil. Science, 1968, 160, 432-433.

SIEGEL, S. Nonparametric statistics for the behavioral sciences. New York: McGraw-Hill, 1956. 